\title{
Investigation of Gold Nanolayer Properties Using X-Ray Reflectometry and Spectroscopic Ellipsometry Methods
}

\author{
I. Stabrawa ${ }^{a, *}$, D. Banaś ${ }^{a, b}$, K. Dworecki ${ }^{a}$, A. Kubala-Kukuśs ${ }^{a, b}$, J. Braziewicz $^{a, b}$, U. \\ MAJEWska $^{a, b}$, J. WUdARCZYK-MoĆKO ${ }^{b}$, M. PAJEK ${ }^{a}$ AND S. GÓŹDŹ ${ }^{b, c}$ \\ ${ }^{a}$ Institute of Physics, Jan Kochanowski University, Świętokrzyska 15, 25-406 Kielce, Poland \\ ${ }^{b}$ Holycross Cancer Center, S. Artwińskiego 3, 25-734 Kielce, Poland \\ ${ }^{c}$ Institute of Public Health, Jan Kochanowski University, al. IX Wieków Kielc 19, 25-317 Kielce, Poland
}

\begin{abstract}
X-ray reflectometry and spectroscopic ellipsometry methods were applied for determination of physical properties of gold nonolayers. The nanolayers were prepared by sputtering of gold on different substrates: borosilicate glass, polished crystalline quartz and crystalline silicon. With X-ray reflectometry technique roughness of the substrates and density, thickness and roughness of gold layers were determined. The results showed decrease in density of the gold layers due to their nanometer thickness and that roughness of the underlayer affects roughness of the gold layer. In addition, thicknesses of the gold layers measured with spectroscopic ellipsometry turned out to be in agreement, within the experimental uncertainty, with results of the X-ray reflectometry method.
\end{abstract}

DOI: 10.12693 /APhysPolA.129.233

PACS: 61.05.cm, 62.23.St, 68.55.-a, 81.07.-b

\section{Introduction}

Interactions of complex biomolecules (DNA, RNA, proteins, antibodies) with solid surfaces, due to their potential application as the biosensor and biomolecular electronics, play very important role in many research areas: biology, biotechnology, chemistry, physics and medicine. Consequently, understanding of the interaction and achieving the ability for controlling of behavior of such biological objects on solid surfaces are the main aims of studies performed nowadays (see e.g. [1]). However, in order to use the biomolecules in functional devices it is essential to immobilize the biomolecules on surfaces in uniform and stable form [2]. One of the often used methods for this purpose is forming a sulphur containing self-assembled monolayer (SAM) on a gold surface using strong chemical bonds between gold and sulphur and next immobilization of sulphur-terminated biomolecules to a gold surface modified by the SAM [1, 2]. In most cases in such studies a gold surface is prepared by deposition of thin gold film, having a thickness from 10 to hundreds of $\mathrm{nm}$, on different substrates. Because properties of such gold films (nanolayers) are significantly different, then thick (bulk) gold [3] precise determination of these properties is necessary for full understanding of a biomolecule-metal surface interaction.

In this study, we use X-ray reflectometry (XRR) and spectroscopic ellipsometry (SE) methods for determination of physical properties of gold nanolayers prepared by sputtering of gold on different substrates. With XRR roughness of a substrates and density, thickness and

*corresponding author; e-mail: ilona.stabrawa@interia.pl roughness of gold layers were obtained. For comparison thickness of the gold layers was additionally determined from SE method.

\section{Experiment}

\subsection{Substrates and Au nanolayers}

The gold layers studied in this work were prepared in Institute of Electronic Materials Technology (ITME, Warsaw, Poland) by sputtering of gold on borosilicate glass (BK7), polished crystalline quartz $\mathrm{SiO}_{2}(100)$ or crystalline silicon. The size and thickness of the substrates were $2 \mathrm{~cm} \times 2 \mathrm{~cm}$ and $1 \mathrm{~mm}$, respectively. Declared thickness of the gold layers was $50 \mathrm{~nm} \pm 2 \mathrm{~nm}$. Before sputtering of gold the substrates were covered by evaporation with about $2 \mathrm{~nm}$ chromium layer in order to improve adhesion of the gold film to the substrate.

\subsection{Diagnostic techniques}

In order to determine properties of the nanolayers we used XRR and SE methods. The XRR method [4], which uses the effect of total external reflection of X-rays, is surface sensitive analytical technique for investigation of the near surface regions of different sample systems including single crystalline, polycrystalline and amorphous samples, polymers, organic samples and fluids. Specular reflection of X-rays on thin film of such samples above critical angle of total reflection gives rise to regular oscillations, so-called Kiessig fringes [5]. These oscillations are result of reflection of X-rays at each layer and further interference of X-rays reflected from different layers of the sample. This phenomenon allows the measurement of thin film thicknesses of single and multilayer systems in the range from $0.1 \mathrm{~nm}$ to $1000 \mathrm{~nm}$, depending on the analytical instrument used. A full reflectometry pattern obtained for the reflection angles from $0^{\circ}$ to about $5^{\circ}$ 
allows modelling density profiles of near surface regions and roughness of surfaces and substrates.

In this work XRR measurements were performed with PANalytical X'Pert PRO MPD diffractometer equipped with $\mathrm{Cu}$-anode $\mathrm{X}$-ray tube and configured for reflectometry with parallel beam. In the incident beam $\mathrm{Cu}$-X-ray path $\mathrm{W} / \mathrm{Si}$ parabolic mirror with $1 / 8^{\circ}$ divergence slit and $1.4 \mathrm{~cm}$ mask was mounted, while in the reflected beam path parallel plate collimator $\left(0.18^{\circ}\right)$ and $\mathrm{X}^{\prime}$ Celerator detector. The angle of reflection $(\theta)$ (between direction of the incoming beam and sample surface) was changed from 0 to $2.5^{\circ}$ with step of $0.005^{\circ}$ (time per step was $8 \mathrm{~s}$ ). As a result time of one measurement was about $1 \mathrm{~h}$.

Thicknesses of the gold layers determined from fitting of an experimental reflectivity pattern were compared with the results obtained with SE method. The SE is non-invasive and non-destructive technique which measures the changes in the state of polarization of light upon reflection from a surface [6]. The polarization change is represented as an amplitude ratio $\Psi$, and the phase difference $\Delta$. The measured parameters depends on optical properties and thickness of individual materials and, as a result, ellipsometry is primarily used to determine film thickness and optical constants [7].

The SE measurement were performed using Sentech SE 800 PV spectroscopic ellipsometer from Sentech Instruments GmbH. This ellipsometer is based on Step Scan Analyzer measurement mode, wide spectral range compensator, and thermoelectrically cooled, high sensitive CCD based multiplex detection, dedicated to fast and accurate measurements of refractive index, absorption and film thickness [8]. During measurements, discussed in this article, the light spot was $2 \mathrm{~mm}$ in diameter and the incident angle was set to $70^{\circ}$. The measurements were performed in the wavelength $(\lambda)$ range from 400 to $800 \mathrm{~nm}$ with sampling step $1 \mathrm{~nm}$.

\section{Results and discussion}

In order to determine the crystal structure of the gold layers X-ray diffraction measurements using X'Pert Pro MPD diffractometer were performed. In Fig. 1, as an example, diffraction pattern measured for $50 \mathrm{~nm}$ gold layer deposited on the quartz substrate is shown. In this figure, diffraction peaks of gold, chromium and quartz substrate are identified and marked. The measurements showed that the gold layers have polycrystalline structure with preferred orientation of grains in the (111) direction.

The reflectivity patterns measured for $50 \mathrm{~nm}$ gold layer deposited on $\mathrm{BK} 7, \mathrm{SiO}_{2}$ and $\mathrm{Si}$ with XRR method are shown in Fig. 2. Density of the layers forming the sample, their thickness and roughness can be obtained by fitting a modelled theoretical curve to the experimental reflectivity pattern. In Fig. 2 results of fitting of the measured reflectivity patterns performed with X'Pert Reflectivity 1.3a (PANalytical) program are presented. This simulation program bases on the Parratt formalism for reflectivity [4]. The key parameters used in the equa-



Fig. 1. Diffraction pattern obtained for $50 \mathrm{~nm}$ gold layer deposited on the quartz substrate.

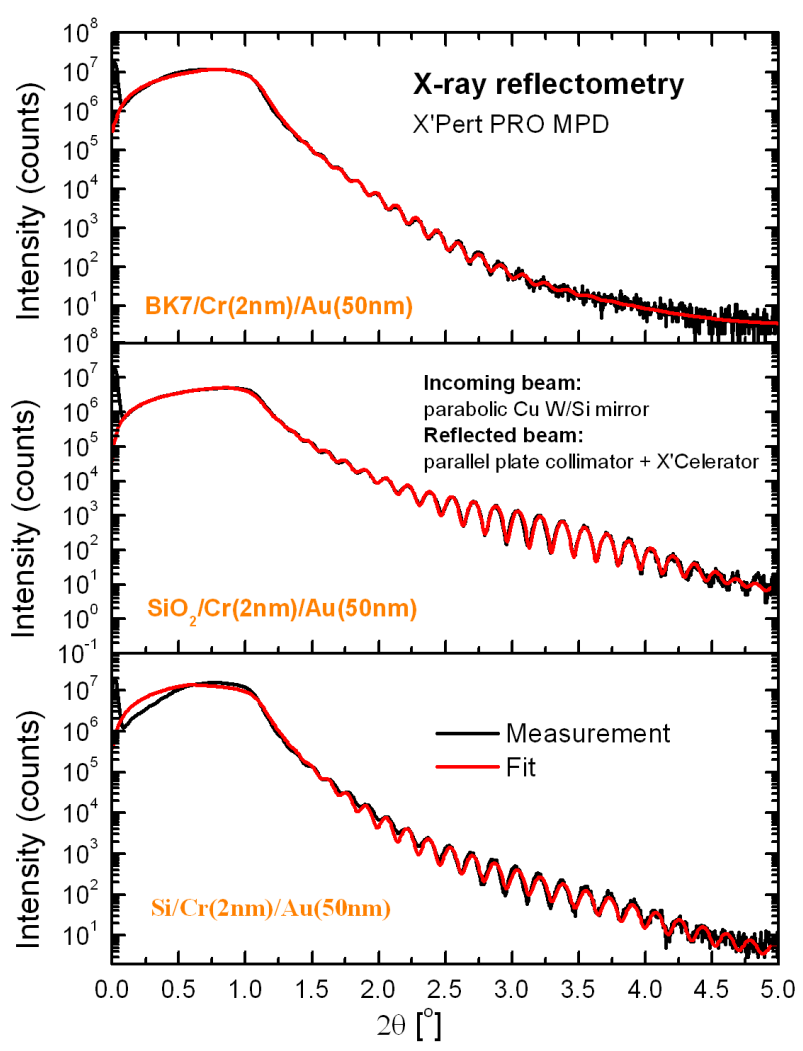

Fig. 2. Reflectometry patterns (black lines) measured for the gold layers deposited on different substrates: borosilicate glass (BK7), polished quartz $\left(\mathrm{SiO}_{2}\right)$ and crystalline silicon ( $\mathrm{Si}$ ) compared with theoretical curves (red lines) obtained using X'Pert Reflectivity 1.3a software (PANalytical). 
tion are materials parameters, which define the refractive index and linear absorption as a function of depth, and instrumental parameters, which define the incident beam characteristics. The refractive index is a function of the wavelength of the radiation and the electron density of the material. During the simulations, for each angle of incidence, the relative reflected intensity is calculated following a recursive formula which combines the reflected and transmitted amplitudes layer-by-layer throughout the whole sample depth [9]. In Fig. 2 the experimental reflectivity patterns are compared with, first modelled, and next fitted to the experimental data, theoretical reflectometry patterns. The very good agreement between the experimental patterns and modelled theoretical curves allows to determine roughness of the substrate, and density, thickness and roughness of chromium and gold layers.

The results are summarized in Table I. Analysing the results in the table it can be seen that obtained thickness of the gold layers agrees within the uncertainties, with the thickness declared ( $50 \pm 2 \mathrm{~nm})$ by producer of the samples. Chromium layers are slightly thicker than declared. Densities of the $2 \mathrm{~nm}$ chromium layers, obtained from the fit, surprisingly agree within the experimental uncertainties with the value for bulk chromium $\left(7.19 \mathrm{~g} / \mathrm{cm}^{3}\right)$. Usually densities of such thin nanolayers significantly differ from the bulk density but nanolayers densities could also depend on the deposition process and possible inhomogeneity of the layers. Fitted densities of gold layers are lower than density of the bulk gold $\left(19.3 \mathrm{~g} / \mathrm{cm}^{3}\right)$. The reason of this is nm thickness of the layers. For gold layers thinner than $100 \mathrm{~nm}$ density begins to decrease and reaches a value of about $15 \mathrm{~g} / \mathrm{cm}^{3}$ for thickness of $10 \mathrm{~nm} \mathrm{[3].} \mathrm{Our}$ results fairly well agree with results presented by Siegel et al. [3]. They estimated that density of $50 \mathrm{~nm}$ gold layer is in the order of $18 \pm 0.5 \mathrm{~g} / \mathrm{cm}^{3}$. Analysing roughnesses presented in Table I one can see that the lowest roughness $(0.81 \mathrm{~nm})$ of the gold layer is obtained for gold deposited on polished quartz $\left(\mathrm{SiO}_{2}\right)$. It is clearly connected with low roughness of the substrate. On this basis it can be concluded that roughness of the substrate affects thickness of the deposited nanolayers. This conclusion agrees with the results of Chalupka et al. [10]. They showed, on gold layers example, that morphology of substrate affects not only speed of the layer growth but also layer thickness, roughness and stability [10].

In Fig. 3, as an example of data obtained with SE method, the $\Psi$ and $\Delta$ curves measured for $50 \mathrm{~nm}$ gold layer deposited on crystalline silicon substrate are presented. In this figure the measured $\Psi$ and $\Delta$ curves are compared with modelled and fitted theoretical curves. The curves were simultaneously fitted to the experimental data with SpectraRay/3 software (Sentech). Performing the fit we found that the influence of the $\mathrm{Cr}$ layer $(2-6 \mathrm{~nm})$ on the determined thickness of the Au layer is negligible, within the fit uncertainty. Obtained good agreement between the modelled curves and experimental data allows to determine thickness of the gold layers.
TABLE I

Roughness of the substrate, and density, thickness and roughness of chromium and gold layers obtained by fitting experimental data with modelled theoretical curves for gold layer deposited on BK7 substrate, polished quartz and crystalline silicon.

\begin{tabular}{c|c|c|c}
\hline \hline layer & density $\left[\mathrm{g} / \mathrm{cm}^{3}\right]$ & thickness $[\mathrm{nm}]$ & roughness [nm] \\
\hline \multicolumn{4}{c}{ BK 7 substrate } \\
\hline $\mathrm{Au}$ & $18.3 \pm 0.2$ & $50.6 \pm 0.9$ & $1.22 \pm 0.01$ \\
$\mathrm{Cr}$ & $9.37 \pm 2.5$ & $3.38 \pm 0.47$ & $1.29 \pm 0.20$ \\
$\mathrm{BK} 7$ & 2.20 & 1000000 & $1.38 \pm 0.26$ \\
\hline \multicolumn{4}{c}{ polished quartz $\left(\mathrm{SiO}_{2}\right)$} \\
\hline $\mathrm{Au}$ & $18.8 \pm 0.1$ & $48.4 \pm 0.1$ & $0.80 \pm 0.01$ \\
$\mathrm{Cr}$ & $7.01 \pm 0.57$ & $2.79 \pm 0.03$ & $0.72 \pm 0.03$ \\
$\mathrm{SiO}_{2}$ & 2.64 & 1000000 & $0.56 \pm 0.05$ \\
\hline \multicolumn{4}{c}{ crystalline silicon $(\mathrm{Si})$} \\
\hline $\mathrm{Au}$ & $18.3 \pm 0.2$ & $48.0 \pm 0.3$ & $1.56 \pm 0.06$ \\
$\mathrm{Cr}$ & $9.01 \pm 1.4$ & $2.54 \pm 0.7$ & $0.87 \pm 0.05$ \\
$\mathrm{Si}$ & 2.33 & 1000000 & $1.31 \pm 0.35$
\end{tabular}

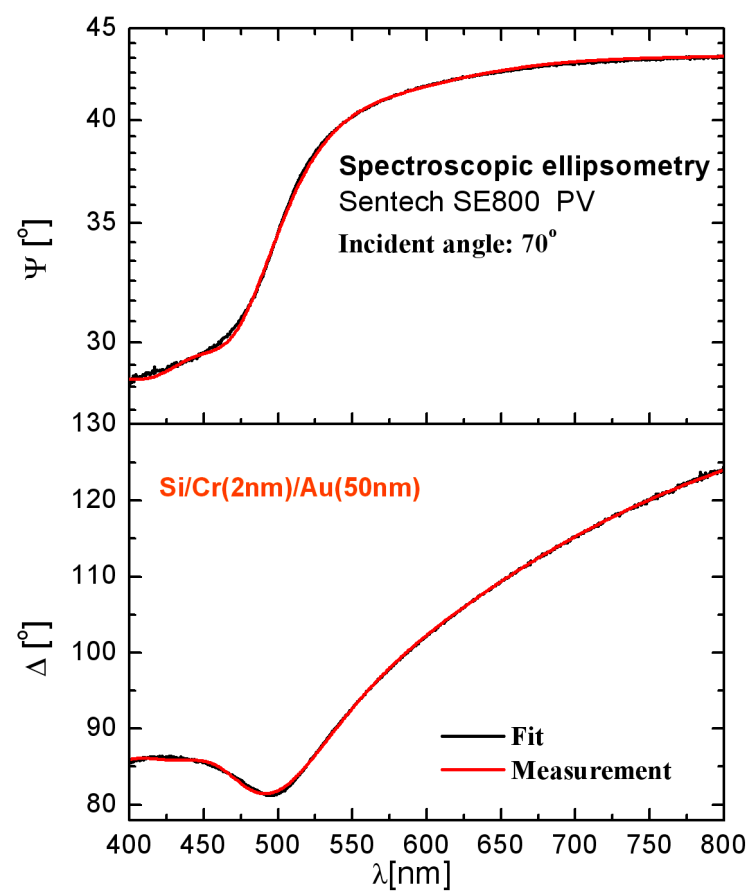

Fig. 3. $\Psi$ and $\Delta$ curves measured with SE method (black lines) for gold layer deposited on crystalline silicon compared with theoretical curves (red lines) obtained using SpectraRay/3 software (Sentech).

The results are summarized in Table II and compared with the thicknesses extracted from XRR measurement.

Comparing the results presented in Table II one can see good agreement between both methods. The attention draws two effects: large uncertainty obtained with XRR technique for glass substrate and significantly higher thickness obtained for $\mathrm{SiO}_{2}$ and $\mathrm{Si}$ substrates with $\mathrm{SE}$ technique. The reason for the first effect is not clear 
TABLE II

Thicknesses of gold layers obtained from SE measurement compared with the results of XRR method.

\begin{tabular}{c|c|c}
\hline \hline substrate & thickness XRR [nm] & thickness SE [nm] \\
\hline $\mathrm{BK} 7$ & $50.6 \pm 0.9$ & $49.8 \pm 0.3$ \\
$\mathrm{SiO}_{2}$ & $48.4 \pm 0.1$ & $49.6 \pm 0.3$ \\
$\mathrm{Si}$ & $48.0 \pm 0.3$ & $49.6 \pm 0.2$
\end{tabular}

at the moment and needs further investigation. The second effect was already observed. Performed systematic studies showed that spectroscopic ellipsometry results indicate systematically higher film thickness as compared to XRR measurements [11]. The probable reason for that is influence of the substrate.

\section{Conclusions}

In this study XRR and SE techniques were applied for determination of physical properties of gold nanolayers prepared by sputtering of gold on different substrates: borosilicate glass, polished quartz, and crystalline silicon. An important advantage of spectroscopic ellipsometry is much easier measurement, thus this method is ideal for the rapid estimation of the thickness of the layer. In reflectometry, time-consuming aligning is necessary due to very low angles used in the measurement. In this paper, roughness of the substrates and density, thickness and roughness of gold layers were determined using XRR technique. Additionally, thickness of the gold layers were determined from SE method. We conclude that thickness of the gold layers determined with SE are slightly higher for $\mathrm{SiO}_{2}$ and $\mathrm{Si}$ substrates then obtained using the XRR method. Moreover, the results showed that density of the layers is lower than the density of the bulk gold, due to their nanometer thickness, and roughness of the underlayer affects significantly roughness of the gold layer.
In the future we plan to use XRR and SE techniques to study gold layers prepared with different techniques and with different thicknesses. We would also like to perform precise measurements of the samples structure using grazing incidence X-ray diffraction (GIXRD) method and photoelectron spectroscopy (XPS) technique.

\section{Acknowledgments}

The equipment was purchased thanks to the financial support of the European Regional Development Fund in the framework of the Polish Innovative Economy Operational Program (contract no. WNP-POIG.02.02.00-26023/08).

\section{References}

[1] S.K. Arya, P.R. Solanki, M. Datta, B.D. Malhotra, Biosens. Bioelectron. 24, 2810 (2009).

[2] M. Honda, Y. Baba, T. Sekiguchi, I. Shimoyama, N. Hirao, Thin Solid Films 556, 307 (2014).

[3] J. Siegel, O. Lyutakov, V. Rybka, Z. Kolska, V. Svorcik, Nanoscale Res. Lett. 6, 96 (2011).

[4] L.G. Parratt, Phys. Rev. 95, 359 (1954).

[5] H. Kiessig, Ann. Phys. 10, 769 (1931).

[6] D. Gonçalves, E.A. Irene, Quim. Nova 25, 794 (2002).

[7] J. Gleńska-Olender, K. Dworecki, S. Sęk, M. Kwinkowski, W. Kaca, Thin Solid Films 548, 343 (2013).

[8] SE 800 PV manual, Sentech.

[9] X'Pert Reflectivity manual (PANalytical B.V).

[10] A. Chaloupka, P. Šimek, P. Šutta, V. Švorčik, Mater. Lett. 64, 1316 (2010).

[11] S. Kohli, Ch.D. Rithner, P.K. Dorhout, Rev. Sci. Instrum. 76, 023906 (2005). 